\title{
Die Zukunft des Öffentlichen Gesundheitsdienstes gestalten
}

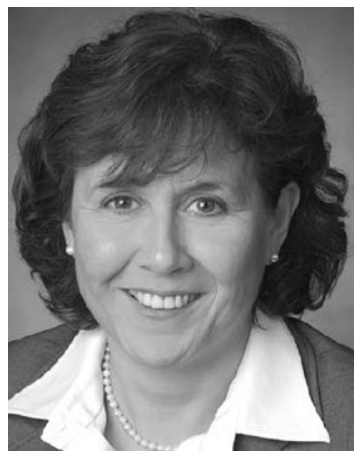

Dr. Ute Teichert
Bibliografie

DOI http://dx.doi.org/ 10.1055/s-0042-104580 Gesundheitswesen 2016; 78: 194

(c) Georg Thieme Verlag KG Stuttgart · New York ISSN 0941-3790

Korrespondenzadresse

Dr. Ute Teichert

Leiterin der Akademie für öffentliches Gesundheitswesen Kanzlerstraße 4 40472 Düsseldorf teichert@akademie-oegw.de
Sehr geehrte Damen und Herren,

liebe Kolleginnen und Kollegen,

Sie besuchen in diesen Tagen den 66. Wissenschaftlichen Kongress der Bundesverbände der Ärztinnen und Ärzte sowie der Zahnärzte des Öffentlichen Gesundheitsdienstes. Wir freuen uns sehr, Sie in diesem Jahr vom 28.-30. April 2016 in Reutlingen zum fachlichen Austausch und der Diskussion neuester Erkenntnisse aus Forschung und Praxis, begrüßen zu dürfen.

Aktuell wie seit Jahrzehnten nicht mehr steht der Öffentliche Gesundheitsdienst derzeit im Blickpunkt der Politik. Im Zusammenhang mit der medizinischen Versorgung von Flüchtlingen gibt es praktisch keinen ÖGD-Bereich, der derzeit nicht gefordert ist und an den die Politiker des Bund, der Ländern und Gemeinden Ansprüche stellt - mit allen Gegensätzen, Zweifeln und Fragen für die Zukunft. Um Schlussfolgerungen für die Zukunft ziehen zu können, müssen wir die Gegenwart verstehen und Erkenntnisse aus den Erfahrungen der Vergangenheit ableiten. Was liegt da näher als auf unserem wissenschaftlichen Kongress eine Rückschau zu halten und die Gegenwart zu beleuchten, um aus unserer derzeitigen Arbeit neue Perspektiven für die Zukunft zu entwickeln. Den Kongress 2016 haben wir daher unter das Thema „Öffentliche Gesundheit im Spiegel der Zeit" gestellt. In der Rückschau setzen wir uns mit der Vergangenheit des Öffentlichen Gesundheitsdienstes, in der Zeit des Nationalsozialistischen Terror-Regimes, auseinander. Insbesondere wie der nationalsozialistische Staat mit seinem kommunal strukturierten und von verschiedensten Einrichtungen getragenen Öffentlichen Gesundheitswesen seit 1935 seine Ideologien, bevölkerungspolitischen Vorstellungen und Ziele umgesetzt hat. Das Forschungsprojekt von Prof. Sabine Schleiermacher wird durch den Bundesverband der Ärzte und Ärztinnen des öffentlichen Gesundheitsdienstes e.V. initiiert und begleitet, das Bundesministerium für Gesundheit fördert das Projekt finanziell.

Die bundesweite Bereitschaft zur ehrenamtlichen Tätigkeit, in der Versorgung von Flüchtlingen, ist ein Beweis, der in Deutschland vorherrschenden Willkommenskultur. Allerdings ist eine medizinische Versorgung von Flüchtlingen durch ehrenamtliche Ärztinnen und Ärzte nicht nachhaltig und kann keine Dauerlösung sein. Wie die Versorgung der großen Zahl an Flüchtlingen sichergestellt und finanziert werden kann, wirft viele Fragen auf. Der ÖGD ist mit seinen begrenzten finanziellen und personellen Ressourcen in der medizinischen Versorgung von Flüchtlingen bisher ein engagierter und verlässlicher
Partner. Wie es jedoch mit dem durch jahrelangen Stellenabbau und personell deutlichen geschwächten ÖGD zukünftig weitergeht, ist nicht sicher. Auf die Fragen wie Gesundheitsämter am Limit zukünftig zur Integration von Flüchtlingen beitragen, oder gemeindenahe Versorgung von Traumatisierten bewerkstelligen sollen, sind klare Antworten gefordert. Gesundheitspolitische Lippenbekenntnisse über den hohen Stellenwert des ÖGD im deutschen Gesundheitssystem reichen nicht mehr aus.

Wichtig ist, dass dabei die Besonderheit des ÖGD und die Fachexpertise in die aktuelle politische Diskussion eingebracht werden. Insbesondere die Erfahrungen und Expertisen in der direkten ärztlichen Versorgung von Flüchtlingen auf kommunaler Ebene sollten wir offensiv nutzen, um den ÖGD neu auszurichten. Hier besteht eine großartige Möglichkeit sich in der Bevölkerung, der Politik und der Ärzteschaft eine neue Attraktivität zu erarbeiten.

Das Thema Flüchtlinge wird für die Gesundheitsämter bundesweit weiter eine zentrale Arbeitsaufgabe sein. Wir sollten diese Chance auf allen Ebenen nutzen, um zusätzliche personelle und finanzielle Ressourcen für den ÖGD zu gewinnen. Die Anzahl der erweiterten Aufgaben traf bereits auf einen ÖGD, der seit Jahren in allen Bereichen am Limit ist. Es ist Allen klar, dass die derzeitigen Herausforderungen nicht ohne Personalaufstockung bewältig werden können. Erste Maßnahmen einer personellen Verstärkung in einzelnen Bundesländern sind ermutigend.

In diesem Zusammenhang ist aber auch - WIEDER - auf eines der größten Probleme des ÖGD aufmerksam zu machen: Nachwuchsmangel. Mit der praktischen ärztlichen Versorgung von Flüchtlingen hat der ÖGD eine einzigartige Chance sich eine neue Attraktivität bei approbierten Ärztinnen und Ärzten und bei Medizinstudierenden zu erarbeiten. Wenn wir diese Verantwortung gemeinsam mit medizinischen Fakultäten annehmen, werden die vielfältigen attraktiven Aufgaben des ÖGD von jungen Ärztinnen und Ärzten besser wahrgenommen.

Abschließend wünsche ich Ihnen beim Besuch der vielfältigen Vorträge, Podiumsdiskussionen und Workshops des Kongress viel Freude. Diese Beiträge bieten nicht nur einen einzigartigen Einblick in die Vielfalt der Tätigkeiten und Chancen der Gesundheitsämter vor Ort, sie zeigen auch die mannigfaltigen Möglichkeiten empirischer Forschung im ÖGD.

Mit freundlichen kollegialen Grüßen

Ihre

Dr. Ute Teichert 